\title{
A Large, Deep Survey of the Taurus Dark Clouds
}

\author{
S. L. Osborne, R. F. Jameson, and P. D. Dobbie \\ Department of Physics and Astronomy, University of Leicester, \\ University Road, Leicester, LE1 7RH, England \\ E. L. Martín \\ Institute for Astronomy, University of Hawaii, 2680 Woodlawn Drive, \\ Honolulu, HI 96822, USA
}

\begin{abstract}
Preliminary results from a deep R, I and Z band survey of $\sim 6$ square degrees of the Taurus Dark Cloud region are presented. 186 potential brown dwarfs have been unearthed, with seven having follow-up spectroscopic data. The spectra reveal three mid/late-type $\mathrm{M}$ dwarfs, of which two show weak $\mathrm{H} \alpha$ emission. If these objects are members of the TDC region, theoretical models suggest masses in the range $10-20 \mathrm{MJ}_{\mathrm{J}}$.
\end{abstract}

\section{Introduction}

Star forming regions (SFRs) are ideal sites in which to search for brown dwarf members because their young age means that these are relatively bright. Hence, very low mass objects $\left(\mathrm{M} \sim 10 \mathrm{M}_{\mathrm{J}} ; \mathrm{M}_{\mathrm{J}}=1\right.$ Jupiter mass) can potentially be observed with only $2 \mathrm{~m}$ class telescopes. Furthermore, the members of an SFR have a common distance and metallicity, and are approximately of the same age, making the transition between theory and observation relatively straightforward. Hence the Taurus Dark Clouds (TDC), which lie at a distance of $140 \mathrm{pc}$ and have a typical age of $4 \mathrm{Myr}$ (Palla \& Stahler 2000), are a very interesting region to study, as shown by Martín et al. (2001).

\section{Observations and Results}

A far optical R, I and Z band survey of 5.65 square degrees of the TDC was undertaken in December 2000 with the Wide Field Camera (WFC) on the Isaac Newton Telescope (INT) at the Observatorio del Roque de los Muchachos, La Palma. The survey is complete to $\mathrm{I}_{\mathrm{WFC}} \sim 21.5$, and has a limiting magnitude of $\mathrm{I}_{\mathrm{WFC}} \sim 23$. Potential substellar members of the TDC were identified using two colour-magnitude diagrams; R vs R-I and I vs I-Z. Using this dual selection process, 186 brown dwarf candidates have been found.

Low resolution spectra of seven of these candidates were obtained in November 2001 with the Echellette Spectrograph and Imager (ESI) on the Keck II Telescope at Mauna Kea, Hawaii. These spectra reveal three mid/late-type M dwarfs (see Figure 1), which is in agreement with their spectral types inferred 


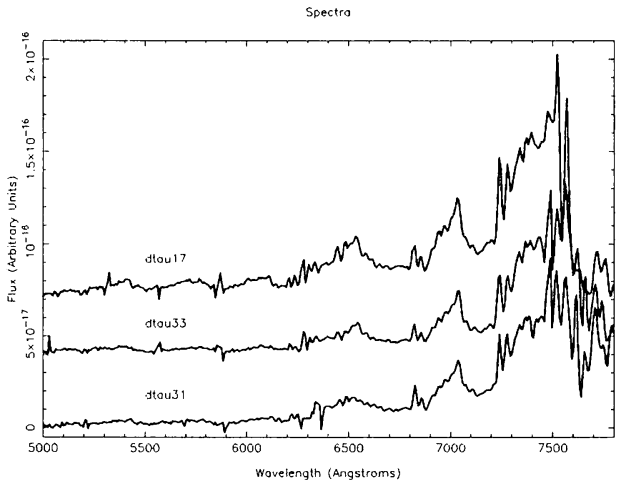

Figure 1. The spectra of the three TDC mid/late-type $\mathrm{M}$ dwarfs.

from their photometric colours. Hereafter, we refer to these objects as dtau17, dtau31 and dtau33. The spectra show strong $\mathrm{TiO}$ absorption bands, indicating that these objects are cool. In addition, the spectra of dtau17 and dtau33 hint at weak $\mathrm{H} \alpha$ emission, indicating that these objects may be young. We note that the equivalent widths (EWs), $5.3 \pm 0.1 \AA$ for dtau17 and $2.7 \pm 0.1 \AA$ for dtau33, are somewhat lower than those measured for other Taurus brown dwarfs (Martín et al. 2001), but are comparable to the EWs derived for some Pleiades brown dwarfs (Martín et al. 1996). The other four spectra indicate reddened background stars of earlier spectral type.

\section{Conclusions}

In a recent survey of $\sim 6$ square degrees of the Taurus Dark Clouds we have found 186 candidate substellar members. We have obtained spectra for seven of these candidates, revealing three potential brown dwarfs. The NEXTGEN models of Baraffe et al. (2002) and the evolutionary models of Burrows et al. (1997) suggest that these objects have masses in the range $10-20 \mathrm{M}_{\mathrm{J}}$, assuming that the age of the TDC region is $3 \mathrm{Myr}$. If these objects are confirmed as definite TDC members and the success rate of $z 25 \%$ is maintained, then we could unearth $\sim 50$ more brown dwarfs in this region. Given the modest number of more massive members in this region, these data may indicate that the IMF of the Taurus SFR is different to that of the field or open clusters, e.g.,. the Pleiades.

\section{References}

Baraffe, I., Chabrier, G., Allard, F., \& Hauschildt, P.H. 2002, A\&A, 382, 563

Burrows, A., et al. 1997, ApJ, 491, 856

Martín, E.L., Rebolo, R., \& Zapatero Osorio, M.R. 1996, ApJ, 469, 706

Martín, E.L., et al. 2001, ApJ, 561, L195

Palla, F., \& Stahler, S.W. 2000, ApJ, 540, 255 